\title{
Nature and Management of Penetrating Spinal Cord Missile Injuries At Shree Birendra Hospital, Chhauni form November 2001 to November 2004
}

Sharma AP $\star$

\section{Introduction}

Spinal cord injury may be defined as an insult to the spinal cord that partially or completely compromises the main functions of the cord. The current conflict with Maoist in Nepal has increased the firearms related violence and subsequently penetrating missile injuries to the spinal cord. The number of missile injuries to the spinal cord in Royal Nepal Army personnel has increased from November 2001, when the Maoist for the first time, attacked the Army Barrack in Dang on 23rd November 2001. The optimal treatment of these injuries remains controversial. Some authors recommend surgery in most cases, whereas others generally advocate a non-surgical approach. The management controversy is further complicated by the fact that regardless of the treatment administered, patients with incomplete deficit often experience neurological improvement, and patients with cord trans-section rarely show more than a marginal recovery. The potential complications of surgery for missile injuries of the spinal cord are also an important consideration. It is thus important that the treating surgeon understand the exact role and scope of surgical intervention, so that neurological function can be preserved and further deterioration recognized and treated. We analyzed the hospital records of 1750 casualties, admitted from Nov.2001 to Nov.2004 at Shree Birendra Hospital and found 19 cases of missile injuries to the spinal cord. We share our experience of 19 cases of missile injuries managed at Shree Birendra Hospital by using a policy of selection for surgery.

\section{Clinical Material and Method}

Between November 2001 and November 2004, 19 patients (18 males and 1 female) with missile injuries to the spinal cord underwent treatment at

Dr. Achyut Prasad Sharma, FRCS (ED)

Col., Head of the Department of Surgery,

Shree Birendra Hospital, Chhauni, Kathmandu, Nepal.
Shree Birendra Hospital. Of these 19 patients, 13 were army personnel, 4 civil patients ( 2 adult male, 1 female and 1 male student) and 2 Armed Police Force sustained missile injury to the spinal cord. Most of the patients sustained bullet injuries either by Pistol or by rifle but few patients had splinter injuries (exploding shrapnel from shells, grenades, improvised explosive device, pellets from handguns etc) and among them 18 patients had skeletal injuries whereas 1 patient had no skeletal injury. The age range was from 14 years to 44 years with a mean age of 27.6 years.

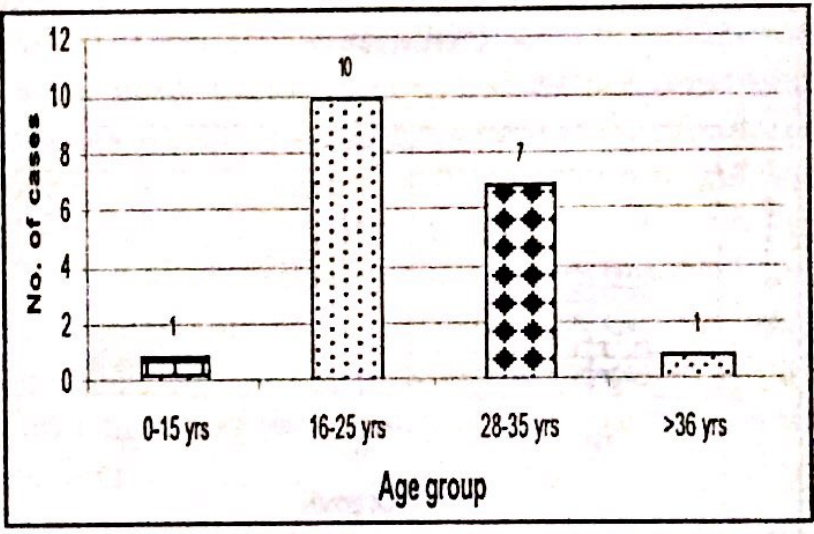

Fig 1: Graph Showing Number of Cases

Associated injuries have been noted in 8 patients (Abdominal visceral injuries in 3 patients, lung parenchyma injuries in 2 patients, vascular and soft tissue injuries neck in 2 patient and brain injury in 1patient).

\section{Clinical evaluation (Triage Room)}

All patients underwent a complete clinical and haemodynamic evaluation on arrival at the Trauma Hall and were neurologically examined once they were haemodynamically stable and the airway was ensured. Most of the injured patients were evacuated from the site of injury to Shree Birendra Hospital by helicopter. Eight patients with associated life-threatening visceral, vascular and brain injuries were taken to operating theatre directly from the Trauma Hall for exploration and 
primary treatment by concerned specialists and neurosurgical procedures were performed as secondary measures but special attention was given for the protection of injured spine and spinal cord and they were admitted in the post operative ward directly form the operation theatre. Detail neurological evaluation of these patients was performed in the post operative ward and necessary imaging studies were performed. Remaining 11 patients had isolated spinal cord injury only and they were haemodynamically stabilized and a thorough neurological evaluation was performed. Any neurological deficit was documented according to American Spinal Injury Association impairment scale. The admission ASIA grades were "A" on 6 patients, no "B" grade, "C" grade on 11 patients and " $D$ " on 2 patients, no "E" grade. After 24 hours of admission, again a neurological evaluation was done and the neurological status recorded.

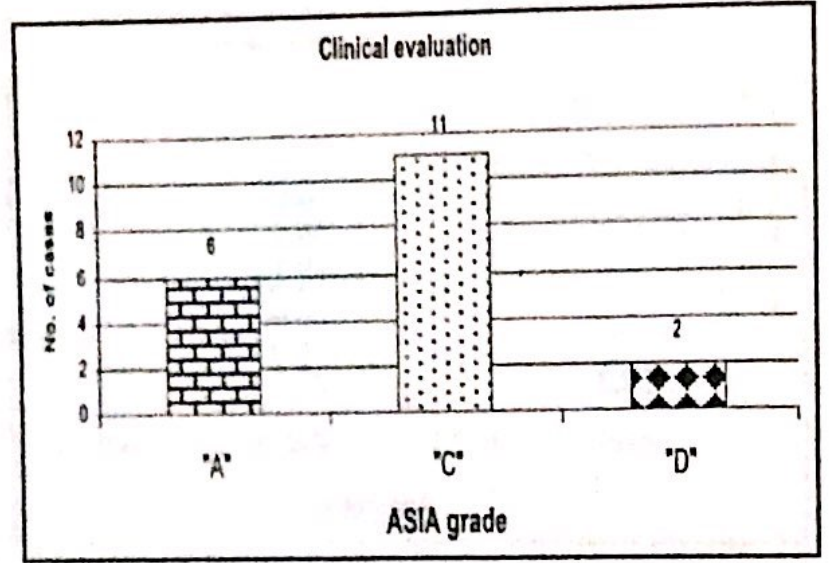

Fig 2: Graph Showing Clinical Evaluation According to ASIA Grade

Following Trauma Hall triage and stabilization, imaging studies were obtained and patients were admitted to Intensive Trauma Care Unit (ITCU). All 19 patients had plain $x$-ray spine, MRI in 14 patients, CT in 5 patients and $C$ T myelography in 1 patient. Imaging studies demonstrated involvement

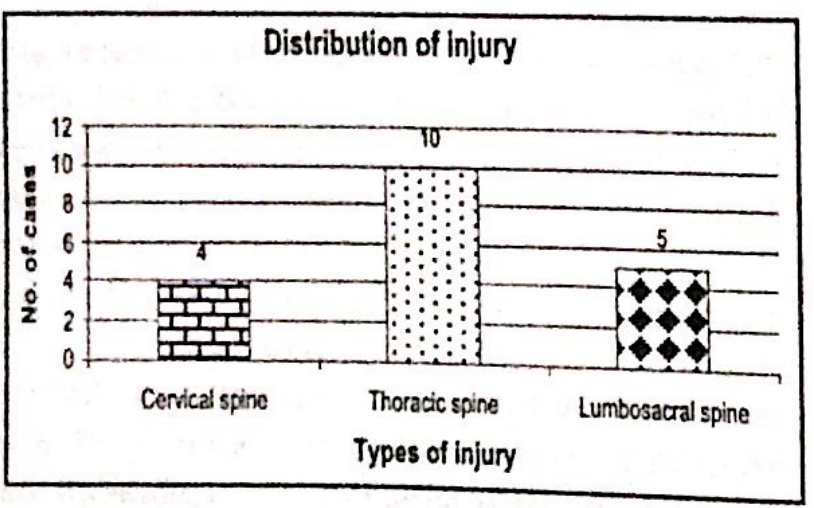

Fig 3: Graph Showing Distribution of Injury of thoracic spine in 10 patients, cervical spine in,
patients and lumbosacral spine in 5 patients

These patients underwent a standard medice management protocol initially, that incluge
intravenous fluids, antibiotics, bladde,
catheterization, care of skin and bowels catheterization, care of skin and bowels. High methylprednisolone injection was given only selected patients who arrived within 8 hours of the injury. Baseline laboratory investigations and $\mathrm{cros}_{\mathrm{n}}$ matching of necessary amount of blood performed.

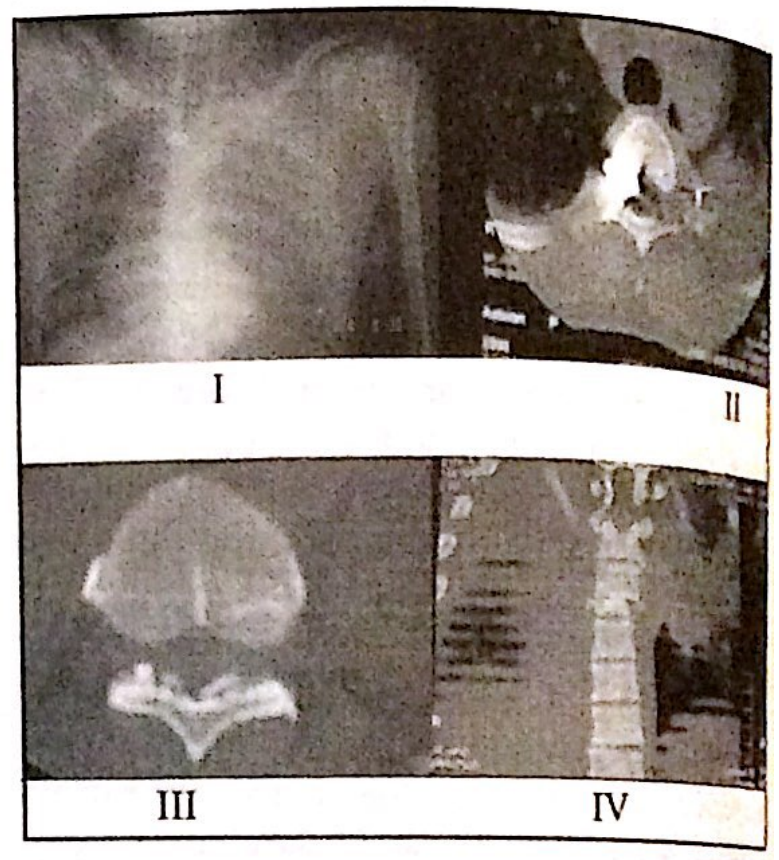

\section{Neurosurgical procedure}

All patients with incomplete cervical, thoracic and lumbosacral missile injuries with neural element compression underwent a neurosurgical procedure where as patients with complete neurological lesion were not taken for neurosurgical exploration but bullet track wound debridement was performed in all missile injured patients. The purpose of the neurosurgical procedure was to decompress and debride neural tissue, remove any bony or metalli: fragments, re-establish dural continuity, secut? haemostasis and correct spinal stability.

In our series, 8 patients underwent a neurosurgical procedure among them 1 patient had cervical cont injury, 3 patients had thoracic cord injury and patients had cauda equina injury. Spinal fixation with neurosurgical procedure was performed in? patients and 3 patients had only neurosurgist procedures performed on them. Surgiost intervention was performed in 7 ASIA grade "C patients and 1 ASIA grade " $D$ " patient. 


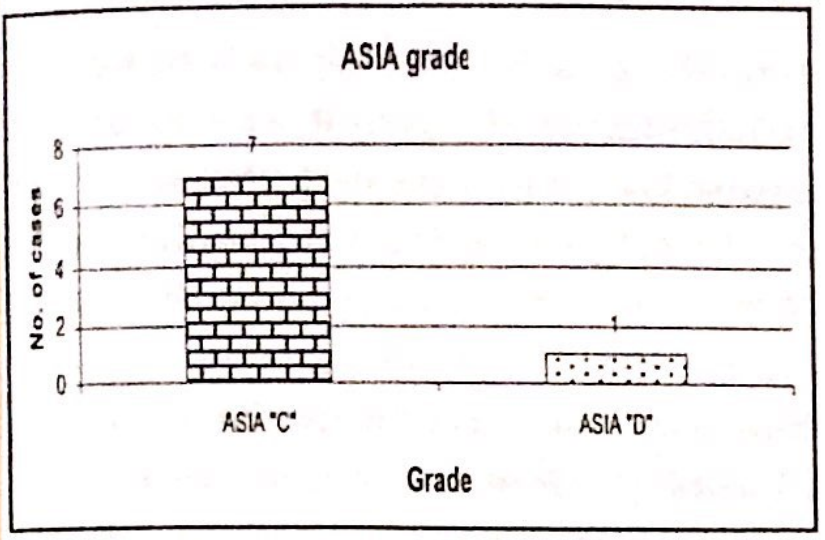

Fig 4: Graph Showing ASIA Grading

The spinal cord exploration was performed posteriorly in lumbar spinal cord, lateral extracavitary or anterior approaches in thoracic cord and anteriorly in cervical spinal cord. Dural closure was performed in all patients and 3 patients needed duroplasty by using lumbodorsal fascia. All patients received antibiotics intravenously for a period of 2 to 6 weeks.

\section{Results}

Of the 19 patients in the study, 8 patients underwent a neurosurgical procedure. There was no improvement in the neurological ASIA grade "A" patients therefore 3 patients remained quadriplegic and 3 patients remained paraplegic. There was distinct improvement in neurological ASIA grade of " $C$ " and " $D$ " patients. All of the patients were discharged from the hospital with useful motor function below the level of lesion. Bladder and bowel dysfunction had also improved. There were no instances of meningitis or CSF fistula. One patient died immediately in the post operative period. Presently, 3 patients with quadriplegia and 3 patients with paraplegia are receiving treatment at Shree Birendra Hospital, Chhauni.

\section{Discussion}

The number of missile injuries to the spinal cord has been increasing since the beginning of the Maoist insurgency, especially from November 2001. The easy availability of handguns, revolvers, shotguns and rifles and the continued and increasing arm struggle in various places around the world demand a renewed and serious interest in and a better understanding of both the ballistic characteristic of missile and the surgical pathology of missile wounds. Missiles may damage the spinal cord by direct laceration, transection, blast wave or by nerve root avulsion leading to intramedullary haematoma. The extent of injury caused by a missile is closely related to the kinetic energy it contains on impact with its target. The final outcome on any case of spinal cord injury depends on the degree of reversible damage to the cord because damage to the neurons and fiber tracts is irreversible and no therapeutic intervention results in spinal cord regeneration and increased functional recovery. A certain proportion of spinal cord injuries are worsened by improper handling of the patient or by the effects of hypo tension or hypoxia on already compromised neural tissue. The velocity of the bullet is the most important determinant of severity of cord injury. The nature of the missile wounds in our series showed that the insurgents are using low velocity weapons. High velocity military weapons produce extensive comminution and wide spread damage due to high-energy transfer. These high velocity missiles may also produce neural damage by transmission of shock waves that penetrate the neural tissue. In such instances, the blast or concussive injuries may have potential for recovery, unlike the caused by direct laceration or transection of the cord. Cauda equina lesions are important injuries which carry a better functional prognosis due to the involvement of the nerve roots and not the spinal cord. At later stages, the cord may be reduced to a fibrotic structure, and the pathological changes may extend above and below the damaged segment. Late deficits are usually a result of the formation of intramedullary cysts or syringomyelic cavities.

In the absence of a therapeutic modality proved to induce regeneration, the best treatment of cord injury is the prevention of secondary injury and begins at the accident scene with personnel trained in safe and expeditious evacuation of these patients to a hospital capable of providing for their definitive care. These casualties are best evacuated by air. Throughout assessment and resuscitation, the spine must be immobilized to avoid secondary injury.

Completeness of injury is based on the presence of detectable function below the level of injury. There is a general agreement among care providers that individuals with no detectable sensory or motor function below the level of injury should be classified as having complete injuries. The Frankel scale has been replaced as the system of classification by the American Spinal Injury Association Impairment scale, which is a 
modification based primarily on the consideration of the S4-S5 requirement for incomplete classification. $A=$ complete: No motor or sensory function is preserved in the sacral segments S4-S5. $\mathrm{B}=$ Incomplete: Sensory but not motor function is preserved below the neurological level and extends through the sacral segments S4-S5. C= Incomplete: Motor function is preserved below the neurological level, and the majority of key muscles below the neurological level have a muscle grade less than 3 . $\mathrm{D}=$ Incomplete: Motor function is preserved below the neurological level, and the majority of key muscles below the neurological level have a muscle grade greater than or equal to 3. E=Normal: Motor and sensory function is normal.

After haemodynamic stabilization and attention to visceral and thoracic injuries, neurological evaluation is carried out and a neurological grade is assigned and imaging studies are performed. All casualties had plain $x$-ray of the spine and CT scan is the principal diagnostic procedure presently and MRI scan is carried out in selected patients. The ability of MRI to directly image the spinal cord not only makes it a more sensitive diagnostic test but theoretically could provide important prognostic clues in the early phases of treatment. MRI is also useful in the demonstration of ligament injuries, and MR angiography has been applied to the diagnosis of cervical vascular injuries.

Following the Trauma Hall emergency room triage and stabilization and physical and neurological examination and imaging studies, patients were taken to the intensive trauma care unit and carefully transferred to a trauma bed using a log-rolling or sliding maneuver. Patient with associated lifethreatening visceral or lung injuries were taken to operating theatre and transferred to the post operative ward from the operating theatre.

Spinal cord injury is a devastating condition both for the patients and also the family, as injury involves not only some degree of paralysis to the individual, but is also commonly accompanied with various degree of physical, emotional and psychosocial barriers. The key to the success of a spinal cord injury care is not necessarily the skill of the physicians or surgeons or the use of experimental drugs, rather, it lies in the development of a multidisciplinary team of surgeons, physicians, nurses, physiotherapist, occupational therapist, prosthetist and orthotist, psychologist, dietician, social worker, vocational counselor, peer counselor, recreational therapist and allied health care professionals committed to and specializing in the treatment of the injury. This team provides a continuity of care that begins at the accident scene and extends through the patient's acute triage, intensive care, and rehabilitations, inpatient programs and beyond to their life long care. Treatment of neurogenic bladder must always be individual and aims at getting patient dry, with complete emptying of a low-pressure bladder in a way that permits to avoid complications and allows a good prognosis. The main possibilities are triggered voiding, intermittent self catheterization plus bladder relaxant drugs or electrotherapy, surgery and condom catheter, indwelling catheter in areflexic detrusor with areflexic sphincter. Bladder expression, external appliances, surgery are amongst the possible ways of treatment. Low cost approach based on proper posture of the patient regular turning at 2 hourly intervals, positioning of pillows, regular care and inspection of skin, care during transfers and patient and care taker education are the most cost effective approaches to pressure ulcer management in the developing country set up.

The traditional approach for the management of missile injuries of the spinal cord has been towards conservatism, arising out of pessimism associated with the poor functional outcome and spinal cord decompression was advised only if there was neurological deterioration during observation. In the military neurosurgical practice, the trend is towards surgical exploration as has been brought out in the 
experiences of the Korean and Vietnam conflicts. During the civil war in Croatia patients were reported to have shown significant neurological improvement and operative mortality of one percent. In our set-up, at the beginning of the insurgency we treated most of the missile injured patients by surgery but presently, the surgical management of missile injuries to the spinal cord is selective because patients with incomplete deficits regardless of the treatment administered often experienced neurological improvement and patients with cord transection rarely show more than a marginal recovery. Therefore patients with complete deficits are generally not surgical candidates unless they have associated radiographic evidence of neural compression, gross contamination or infection and persistent CSF leak, whereas patients with incomplete deficits underwent surgery if they demonstrated a ventral or dorsal compression, partial spinal cord transection, CSF leak and unstable spine and most of the patients with cauda equina lesions underwent surgical intervention. The nature of surgery was debridement, decompression, haemostasis, dural repair and spinal fixation if indicated. The timing of surgery depends upon associated injuries and those cases should be tackled electively once systemic stability is achieved. Neurological deficits without radiographic abnormality were managed with local wound care and supportive measures.

\section{Conclusion}

In recent years, the number of missile injuries to the spinal cord in the military arena has increased. The management of such injuries starts at accident site with proper immobilization, resuscitation and evacuation to central hospital, where definitive treatment is given and rehabilitation programs are started by a team of experts committed to and specializing in the treatment of the spinal cord injuries. Patient needs thorough clinical and neurological evaluation on arrival to the central hospital and necessary imaging studies are obtained and decision is made for surgical or non-surgical management. The role of surgery has yet to be examined by a prospective randomized study as surgery is still debatable in complete neurological deficit. What perhaps focuses the surgical mind is the question "Does this patient need an operation or will the diagnosis suggested by the patient's symptoms best be improved by a surgical intervention in the operating room, or is a nonsurgical intervention or conservative approach more applicable to relieve the symptoms and achieve rehabilitation and a normal quality of life?" The abruptness of the surgical question focuses the mind and demands a decision that requires action, which distinguishes the surgical approach. A decision to act is not a process without intellectual input. To make this decision, the surgeon must balance the advantages and disadvantages and the benefits and the risks as well as the wishes of the patient and the patient's relatives. Incomplete injuries and cauda equina lesions constitute definite indications for surgery. The initial neurological grade is the best prognostic indicator. Rehabilitation efforts should be initiated as soon as possible in all patients. The management of these injured patients should be done at trauma centre by multidisciplinary team of surgeons, physicians, nurses and allied health care professionals committed to and specializing in the treatment of this injury which starts from injury site through pre-hospital service, in hospital service and to rehabilitation of the injured patient and continuous beyond to their life-long care.

\section{Reference:}

1. Cheryl A. Muszynski, Raj K, Narayan. Surgical management of penetrating injuries of the spine, in Henry H. Schmidek, William H. Sweet (eds): Operative Neurosurgical techniques; Indications, Methods and Results. 3rd Edition 1995: pp 19711980.

2. Richard C.S. Kerr and James Wilson MacDonald. The spine, Vertebral Column and Spinal Cord in R.C.G. Russel, Norman S. Williams, Cristopher J. K. Bulstrade (eds): 
3. Bailey and Love's Short Practice of Surgery. 24th Edition 2004. Arnold. pp 552-563.

4. Williams, R. H and Rengachary, S. S. Neurosurgery, 2nd Edition. Mc Graw-Hill, New York. 1996

5. B.A. Green, C. David, S. Falcone, N. Razack, K.J. Klose: Spinal cord injury in adult, in Julian R. Youmans (ed): Neurological Surgery. Vol. 3, 1996. W.B. Saunders. Pp 1969-1980.

6. Yashon D, Jane J. A, White R.J: Prognosis and management of spinal cord and cauda equina bullet injuries in sixty-five civilians. J. Neurosurgery. 32:163-170, 1970.

7. Benzel E.C, Hadden J.A, Coleman J.E: Civilian gunshot wounds to the spinal cord and cauda equina. Neurosurgery 20: 281-285, 1987.

8. Miller C.A: Penetrating wounds of the spine in Wilkin's R.H, Rengachary S.S, (eds): Neurosurgery, Vol. 2 New York Mc Graw, 1985. pp 1746-1748.
9. Venger B.H, Simpson R.K, Narayan R.K Neurosurgical intervention in penetrating spinal trauma with associated visceral injury. 1 Neurosurgery 70:514-518.

10. Jacobson S.A, Bass E: Spinal cord injury in Vietnamese Combat. Praplegia 7.263-281,1970.

11. Tindall S, Bierbrauer K: Brain and spinal injuries caused by missiles, in Long DM (ed): Current Therapy in Neurological Surgery, 2nd Edition. Philadelphia, B.C. Decker, 1989, pp 187. 190.

12. Kihtir T, Ivatury R, Simon R, Stalhl W. M; Management of transperitoneal gunshot wounds of the spine. J. Trauma 31: 1579-1583, 1991. 